\title{
Theory of Ignition of a Reactive Solid by Constant Energy Flux
}

\author{
A. LIÑ̂́N \\ Escuela de Ingenieros Aeronáuticos, Ciudad Universitaria, Madrid, and \\ Instituto Nacional de Técnica Aeroespacial, "Esteban Terradas," Madrid \\ AND \\ F. A. WILLIAMS \\ Department of Aerospace and Mechanical Engineering Sciences, University of California, \\ San Diego, La Jolla, California
}

\begin{abstract}
A formula for the ignition time, that Bradley obtained empirically, is derived analytically by a rigorous asymptotic analysis of the limit of large activation energy. Two terms in the expansion must be retained. The second term reveals the existence-just prior to ignition-of a reactive-diffusive zone at the surface of the solid and a transient-diffusive zone in the interior. The analysis also exhibits a universal correction factor for Bradley's formula, of order unity.
\end{abstract}

\section{INTRODUCTION}

Bradley (1970) solved numerically the equations describing heating of a homogeneous, semi-infinite, reactive solid by an energy input of constant rate at the surface. He defined a critical nondimensional heating time for ignition, $\tau_{c}$, as the nondimensional heating time required for the surface temperature to achieve a minimum in an additional time $\tau_{c}$ after cessation of heating. This definition corresponds most closely to the ignition time measured in "go-no-go" experiments. However, Bradley found that over the physically interesting range of parameters, this critical time differed by less than one percent from ignition times obtained from various other reasonable definitions of ignition. Therefore it seems appropriate to attempt to calculate $\tau_{c}$ from a "thermal runaway" condition, without considering cessation of heating.

Bradley found empirically that over the entire range of parameters of physical interest, $\tau_{c}$ is correlated within four percent by the formula

$$
\begin{aligned}
A= & \sqrt{E^{\prime}}\left(\pi \tau_{c}\right)^{-1 / 4}\left(1+2 \sqrt{\tau_{c} / \pi}\right)^{-1} \\
& \exp \left[E^{\prime} /\left(1+2 \sqrt{\tau_{c} / \pi}\right)\right]
\end{aligned}
$$

Here $A$ is a nondimensional parameter involving inversely, the rate of energy absorption and directly the preexponential factor of the Arrhenius reaction rate, and $E^{\prime}$ is the nondimensional activation energy. Figure 13 of Bradley's paper demonstrates clearly the excellent agreement between equation (1) and the results of the numerical integrations. The purpose of the present paper is to derive equation (1) in a logical and analytical manner, by investigating the limit of large activation energy from the viewpoint of matched asymptotic expansions. The study reveals some new physical attributes of the ignition process and also yields a small correction to equation (1).

The derivation in the text is intended to be heuristic in order to emphasize physical aspects of the development. Appendix A contains aspects of the asymptotic analysis that are more rigorous. The reader is referred to Bradley (1970) for discussions of related work, for precise definition of the physical problem and for the basic equations. The notation herein is identical to that of Bradley.

\section{INERT STAGE}

Since Bradley (1970) has shown that for parameter values of practical interest the ignition time is insensitive to the value of the reaction order $a$, we set $a=0$ and ignore reactant depletion, except in Appendix A, where the effect of reactant depletion is assessed. With $a=0$, equations (1), (3), (4) and (5) of Bradley's paper become

$$
\begin{gathered}
\theta_{\tau}=\theta_{\xi \xi}+A \exp \left(-E^{\prime} / \theta\right) \\
\theta_{\xi}(0, \tau)=-1, \quad \theta(\infty, \tau)=\theta(\xi, 0)=1
\end{gathered}
$$

where $\theta$ is the nondimensional temperature, $\tau$ the nondimensional time and $\xi$ the nondimensional distance normal to the surface.

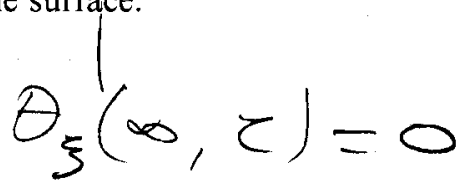


Defining the quantity $\theta_{1}$ by

$$
A=\exp \left(E^{\prime} / \theta_{1}\right)
$$

we assume that $\theta_{1}>1$, since this choice corresponds to the ignition regime analyzed by Bradley. For $0<\theta_{1}<1$, the external heating rate is so small in comparison with the initial chemical heat release rate that a nearly adiabatic and homogeneous explosion occurs (at time $\tau_{I}=\left(A E^{\prime}\right)^{-1} \exp E^{\prime}$ ) instead of ignition. For negative values of $\theta_{1}$, the external heating rate is so large in comparison with the maximum achievable chemical heat release rate that a well-defined ignition phenomenon cannot occur. We shall consider neither of these other two regimes.

Substitution of equation (4) into equation (2) yields

$$
\theta_{r}=\theta_{\xi \xi}+\exp \left[\left(E^{\prime} / \theta_{1}\right)\left(\theta-\theta_{1}\right) / \theta\right],
$$

from which it is seen that in an asymptotic analysis for $E^{\prime} / \theta_{1} \rightarrow \infty$, the reaction-rate term will be exponentially small for $\theta<\theta_{1}$ and exponentially large for $\theta>\theta_{1}$. Thus, to lowest order in the small parameter $\theta_{1} / E^{\prime}$, when $\tau$ is small enough to assure that $0<0_{1}$ everywhere in the solid, then equation (5) reduces to the heat-conduction equation

$$
\theta_{\tau}=\theta_{\xi \xi},
$$

to which the boundary conditions given in equation (3) must be applied. It may be noted from equation (5) that when $\left(\theta-\theta_{1}\right) / \theta$ is negative and of order unity, equation (6) holds to every algebraic order in $\theta_{1} / E^{\prime}$; the first nonvanishing correction in the inert heat-conduction stage is of exponential order in $-\left(E^{\prime} / \theta_{1}\right)\left(\theta_{1}-\theta\right) / \theta$.

The solution for $\theta$ in the early stage of inert heating is well known and will be denoted by $\theta_{I}(\xi, \tau)$, viz.,

$0_{I}=1+2 \sqrt{\tau / \pi} \exp \left(-\xi^{2} / 4 \tau\right)-\xi \operatorname{erfc}(\xi / 2 \sqrt{\tau})$.

Equation (7) shows that $\theta_{I}$ is a monotonically increasing function of $\tau$ and a monotonically decreasing function of $\xi$. Therefore the condition for breakdown of the inert heat-conduction approximation, viz. $\theta_{I}=\theta_{1}$, occurs earliest at the surface of the solid, $\xi=0$. Since equation (5) shows that for $\theta_{I}(0, \tau)>\theta_{1}$ the reaction rate is exponentially large, it is clear that to lowest order in $\theta_{1} / E^{\prime}$, the quantity $\theta_{1}$ represents an ignition temperature, such that thermal runaway occurs when the nondimensional surface temperature reaches $\theta_{1}$. By setting $\theta_{I}=\theta_{1}$ and $\xi=0$ in equation (7), one obtains an expression for the ignition time $\tau_{1}$ in lowest order, viz.,

$$
\theta_{1}=1+2 \sqrt{\tau_{1} / \pi}
$$

Equations (4) and (8) give

$$
A=\exp \left[E^{\prime} /\left(1+2 \sqrt{\tau_{1} / \pi}\right)\right],
$$

which reproduces the major part of equation (I) if we identify $\tau_{1}$ as $\tau_{c}$ to lowest order.

\section{TRANSITION STAGE}

\subsection{Establishment of the problem}

Having obtained the major part of equation (1) from analysis of the lowest order, we can attempt to derive the remaining factors in equation (1) by proceeding to the next higher order. One logical approach to the higher-order analysis is to treat $A$ as a specified constant and to calculate a correction to $\tau_{1}$ for the ignition time. We remark without proof that through a development closely resembling that given below, this approach leads directly to the formula

$$
\tau_{c}=\tau_{1}+\sqrt{\pi \tau_{1}}\left(\theta_{1}^{2} / E^{\prime}\right)\left[b+\ln \left\{\left(\theta_{1}^{2} / E^{\prime}\right) / \sqrt{\pi \tau_{1}}\right\}^{1 / 2}\right],
$$

where to lowest order in $1 / E^{\prime}, b$ is a universal constant of order unity. Although equation (10) with $b=0$ is asymptotically equivalent to equation (1), it is tedious to demonstrate the equivalence explicitly. Since equation (1) is an explicit expression for $A$ in terms of $\tau_{c}$, a more direct approach to the derivation of equation (1) is to treat the ignition time as a specified constant and to calculate the corresponding value of $A$. Such an approach is adopted herein.

Thus, we specify that the nondimensional ignition time will be $\tau_{c}$, and we define a corresponding inert ignition temperature by

$$
\theta_{c}=1+2 \sqrt{\tau_{c} / \pi},
$$

as given by equation (7). Writing equation (2) in the form

$\theta_{\tau}=\theta_{\xi \xi}+A \exp \left(-E^{\prime} / \theta_{c}\right) \exp \left\{\left(E^{\prime} / \theta_{c}\right)\left(\theta-\theta_{c}\right) / \theta\right\}$,

we must solve equation (12) subject to the boundary conditions given in equation (3). Imposing on this solution the ignition condition of thermal runaway at $\tau=\tau_{c}$ will then determine $A$. 
Anticipating that $A \exp \left(-E^{\prime} / \theta_{c}\right)$ will be of algebraic order in our small parameter $\theta_{c} / E^{\prime}$, we obtain the inert heating problem of the previous section for $\theta<\theta_{c}$. To analyze the transition from inert heating to vigorous reaction, we introduce the deviation of the temperature from that corresponding to inert heating as a new dependent variable, viz.,

$$
\Phi=\theta-\theta_{I},
$$

where $\theta_{I}$ is given by equation (7). The problem to be solved for $\Phi$ then takes the form

$$
\begin{aligned}
\Phi_{\tau}= & \Phi_{\xi \xi}+A \exp \left(-E^{\prime} / \theta_{c}\right) \\
& \times \exp \left[\left(E^{\prime} / \theta_{c}\right)\left(\theta_{I}-\theta_{c}+\Phi\right) /\left(\theta_{I}+\Phi\right)\right], \\
& \Phi_{\xi}(0, \tau)=\Phi(\infty, \tau)=\Phi(\xi, 0)=0 .
\end{aligned}
$$

\subsection{The reactive-diffusive zone}

Since $E^{\prime} / \theta_{c}$ is large, departures from inert heating $(\Phi=0)$ occur first at times and positions such that $\theta_{c}-\theta_{I}$ is close to zero. These times will be near $\tau_{c}$, and the temperature gradient in the solid during inert heating will cause the positions to correspond to small values of $\xi$. Therefore to investigate transition to ignition, in equation (14) we can expand $\theta_{I}$ about $\tau=\tau_{c}, \xi=0$. From equation (7), this expansion is found to be

$$
0_{I} \simeq \theta_{c}-\xi+\left(\tau-\tau_{c}\right) / \sqrt{\pi \tau_{c}} .
$$

Substitution of this expression into equation (14), and retention of only the first nonvanishing term in the exponent, yields

$$
\begin{aligned}
\Phi_{\tau}= & \Phi_{\xi \xi}+A \exp \left(-E^{\prime} / \theta_{c}\right) \\
& \times \exp \left\{\left(E^{\prime} / \theta_{c}^{2}\right)\left[\Phi-\xi+\left(\tau-\tau_{c}\right) / \sqrt{\pi \tau_{c}}\right]\right\} .
\end{aligned}
$$

In the reaction zone near the surface of the solid, it is physically clear that during the transition stage, both space and time variations of the inert temperature field must be included. Therefore, in equation (17) we wish to introduce stretched variables such that the $\xi$ term and the $\tau$ term are of the same order in the exponent. For any such stretching, it is seen that in equation (17), $\Phi_{\tau}$ is necessarily of higher order than $\Phi_{\xi \xi}$. Therefore, in the first approximation we neglect $\Phi_{\tau}$ in equation (17). This causes equation (17) to describe a reactivediffusive regime in which transient effects are negligible. Requiring further that the $\Phi$ term remains in the exponent, a condition that is needed to achieve thermal runaway at a finite time, we find that a suitable small translation of the stretched time coordinate removes all parameters from equation (17).

Explicitly, with

and

$$
\psi \equiv\left(E^{\prime} / \theta_{c}^{2}\right) \Phi, \quad x \equiv\left(E^{\prime} / \theta_{c}^{2}\right) \xi
$$

$$
\begin{aligned}
t \equiv\left(E^{\prime} / \theta_{c}^{2}\right)\left(\tau-\tau_{c}\right) / \sqrt{\pi \tau_{c}}+\ln \left(\theta_{c}^{2} / E^{\prime}\right) \\
+\ln \left[A \exp \left(-E^{\prime} / \theta_{c}\right)\right],
\end{aligned}
$$

to lowest order in $\theta_{c}^{2} / E^{\prime}$ equation (17) becomes

$$
\psi_{x x}=-\exp (\psi-x+t),
$$

with boundary and initial conditions

$$
\psi_{x}(0, t)=\psi(\infty, t)=0, \quad v(x,-\infty)=0,
$$

as obtained from equation (15). Since $t$ appears only parametrically in equation (20), no initial conditions may be imposed on it; however, since $t$ may be absorbed into $x$ by introducing the new variable $x-t$, it is clear that the initial condition will be satisfied automatically if the boundary condition at $x=\infty$ is satisfied.

In terms of the variable $F \equiv \psi-x$, equation (20) is $F_{x x}=-e^{F+t}$, whose first integral is $F_{x}^{2}=$ $c-2 e^{F+t}$, where $c$ is an arbitrary constant. The boundary condition on $\psi_{x}$ at $x=0$ then requires $c=1+2 \exp \left(\psi_{0}+t\right)$, where $\psi_{0}$ is the time-dependent value of $\psi$ at $x=0$. However, the boundary conditions at $x=\infty$ cannot be satisfied, because equation (20) has $\psi_{x x}<0$, which requires that for $x>0, \psi_{x}$ must be less than its value at $x=0$, which is zero. This result demonstrates the necessity of introducing an additional spatial region during the transition stage, for large values of $x$, where equation (20) is inapplicable. The surface boundary conditions for this region of large $x$ is the relationship between $\psi$ and its $x$ derivative obtained by evaluating the reactivediffusive solution at $x=\infty$, viz.,

$$
\psi_{x \infty}=1-\left[1+2 \exp \left(\psi_{0}+t\right)\right]^{1 / 2},
$$

where the negative sign has been selected for the square root to satisfy the requirement that $\psi_{x}<0$.

\subsection{The transient diffusive zone}

To analyze the outer region of large $x$, we must return to equation (14) and retain the transient term. To be able to satisfy boundary conditions, the diffusive term should be retained in the outer region. Since time scaling must be the same for both inner and outer regions in the transition stage, we employ $t$ as the time variable and scale $\xi$ to assure that $\Phi_{\tau}$ and $\Phi_{\xi \xi}$ will be of the same order. 
It follows that the appropriate spatial variable is

$$
\eta \equiv \xi \sqrt{E^{\prime} \mid \theta_{c}^{2}}\left(\pi \tau_{c}\right)^{-1 / 4}=x \sqrt{\theta_{c}^{2} / E^{\prime}}\left(\pi \tau_{c}\right)^{-1 / 4} \text {. }
$$

Equation (14) then becomes simply

$$
\psi_{t}=\psi_{\eta \eta}
$$

since the reaction rate is exponentially small, as can be seen, for example, from equation (17), in which the transformations produce the factor $\exp \left(-\eta \sqrt{E^{\prime} / \theta_{c}^{2}}\right)$ in the heat-production term. The initial and boundary conditions for equation (24) become

and

$$
\psi(\eta,-\infty)=\psi(\infty, t)=0
$$

$$
\begin{aligned}
\psi_{\eta}(0, t)= & \sqrt{E^{\prime} / \theta_{c}^{2}}\left(\pi \tau_{c}\right)^{1 / 4} \\
& \times\left\{1-[1+2 \exp \{\psi(0, t)+t\}]^{1 / 2}\right\},
\end{aligned}
$$

the last of which is the matching condition implied by equation (22). These results show that in the transition stage, the regime of large $x$ is a transientdiffusive zone.

For early times, the exponential term in equation (26) is small compared with unity, and a two-term expansion of the square root can be employed. After this expansion is made, an additional small translation in time removes all parameters from the outer problem. Thus, we define

$$
\sigma=t+\ln \left[\sqrt{E^{\prime} / \theta_{e}^{2}}\left(\pi \tau_{c}\right)^{1 / 4}\right]
$$

and obtain the problem

$$
\left.\begin{array}{c}
\psi_{\sigma}=\psi_{\eta \eta}, \quad \psi(\eta,-\infty)=\psi(\infty, \sigma)=0, \\
\psi_{\eta}(0, \sigma)=-\exp [\psi(0, \sigma)+\sigma] .
\end{array}\right\}
$$

In the solution to this well-defined problem, shown in Figure $1, \psi(0, \sigma)$ goes to infinity, i.e., thermal runaway occurs, at a finite value of $\sigma$ of order unity. Let us denote this value of $\sigma$ by $b$. Then, for $\sigma \leqslant b$,

$$
\begin{aligned}
& 2 \exp \{\psi(0, t)+t\} \\
& \leqslant 2 \sqrt{\theta_{c}^{2} / E^{\prime}}\left(\pi \tau_{c}\right)^{-1 / 4} \exp \{\psi(0, \sigma)+b\},
\end{aligned}
$$

from which it is seen that for small values of $\theta_{e}^{2} / E^{\prime}$, the assumption that the square root in equation (26) can be expanded, is indeed justified as an asymptotic parameter expansion in $\theta_{c}^{2} / E^{\prime}$. This completes the analysis of the transition stage.

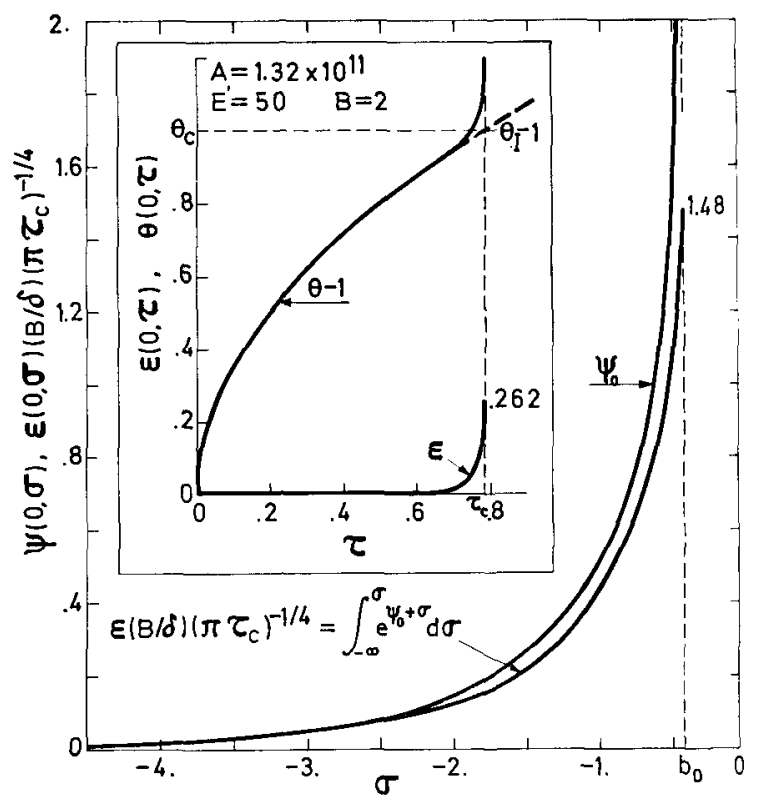

FIG. 1. Results of the numerical integration for the reactant consumption at the surface and for the surface temperature during the transition stage. Also, illustration of the composite expansion for the history of the surface temperature and the surface reactant depletion, in a typical case.

\subsection{History of surface temperature}

Results of the analysis can be illustrated conveniently by the typical surface temperature history sketched in the insert in Figure 1. The surface temperature obtained from the composite expansion is seen from equations (7), (13) and (18) to be

$$
\theta(0, \tau)=1+2 \sqrt{\tau / \pi}+\left(\theta_{\mathrm{c}}^{2} / E^{\prime}\right) \psi(0, \sigma)
$$

in which $\sigma$ is related to $\tau$ through equations (19) and (27). For $\tau<\tau_{c}$, equation (19) shows that $\sigma$ is negative and large in magnitude unless $\tau$ is very close to $\tau_{c}$. For large negative $\sigma$, the solution to equation $(28), \psi(0, \sigma)=e^{\sigma}+e^{2 \sigma} / \sqrt{2}+\cdots$, shows that $\psi$ is very small and that therefore $\theta$ is very close to the inert-heating solution. As $\tau$ approaches $\tau_{c}$, roughly when $\left(\tau_{c}-\tau\right) / \tau_{c}$ becomes of order $\theta_{c} / E^{\prime}$, then $\sigma$ becomes of order unity, and the surface temperature increases above the inertheating result, through a term or order $\theta_{c}^{2} / E^{\prime}$, due to the onset of an appreciable rate of chemical heat release. When $\tau$ reaches $\tau_{\varepsilon}, \psi(0, \sigma)$ and the surface temperature are predicted to be infinite in the present asymptotic analysis, thereby defining thermal runaway. 
Of course, infinite temperatures will not occur at finite times in the exact solution to equations (2) and (3). The infinity is a consequence of the linearization of the exponential, viz. replacing $\exp \left(-E^{\prime} / \theta\right)$ by $\exp \left\{-\left(E^{\prime} / \theta_{c}\right)\left[1-\left(\theta-\theta_{c}\right) / \theta_{c}\right]\right\}$, which is required by the present transition-stage analysis. The fact that the linearization must be performed about $\theta_{c}$ is not essential to this result; linearization about any temperature produces an infinite surface temperature at a finite time, although such a linearization can be justified from the viewpoint of asymptotic theory only for temperatures sufficiently lower than the activation temperature $E^{\prime}$. In the exact solution to equations (2) and (3), the surface temperature grows very rapidly in the vicinity of $\tau=\tau_{c}$, until it reaches a value of the order of $E^{\prime}$, at which point it begins to increase much more slowly, eventually achieving a roughly linear growth with $\tau$, as $\tau \rightarrow \infty$. These later stages are of no physical interest, since reactant depletion will cause equations (2) and (3) to break down before they are reached. To predict the ignition time it is unnecessary to go beyond the transition stage, because the rate of increase of surface temperature becomes so large in this stage that ignition will occur, by any reasonable definition, within a very narrow range of $\tau$ about $\tau_{c}$.

\section{IGNITION TIME}

Since we have shown that thermal runaway, i.e. ignition, occurs at $\sigma=b$, we can trace back the definitions, through equations (11), (19) and (27), to obtain an explicit expression for $A$ in terms of $\tau_{c}$. By definition, in equation (19), $\tau=\tau_{c}$ at ignition. Straightforward substitution yields

$$
\begin{aligned}
A=e^{b} \sqrt{E^{\prime}}\left(\pi \tau_{c}\right)^{-1 / 4} & \left(1+2 \sqrt{\tau_{c} / \pi}\right)^{-1} \\
& \times \exp \left[E^{\prime} /\left(1+2 \sqrt{\tau_{c} / \pi}\right)\right] .
\end{aligned}
$$

Except for the additional correction factor $e^{b}$, equation (29) is identical with equation (1). Equation (28) has been integrated numerically by the method outlined in Appendix B. From this integration it was found that $e^{b}=0.65$. On the $\log$ scale shown in Bradley's Figure 13, this produces a shift of the curves to the right by an amount 0.188 , which is less than twice the thickness of the lines in the figure. Therefore the correlation formula obtained by Bradley is justified rather well from the viewpoint of asymptotic analysis.

\section{CONCLUDING COMMENTS}

The concepts of asymptotic analysis, employed herein, enable one to see quickly how to extend the analysis to more complicated systems and what the nature of the results will be. For example, suppose that two independent reactions were to occur in the solid. It is obvious immediately, from the analysis, that if both reactions are exothermic, then the reaction with the lower ignition time will control the ignition process, and the reaction with the higher ignition time will be entirely negligible in the pre-ignition stages of inert conduction and transition. If a reaction is endothermic, then it alone cannot produce ignition, but it does exhibit a definite time of onset for high activation energies, which is given approximately by equation (1). If this onset time exceeds the ignition time of a second, exothermic reaction, then the endothermic reaction is entirely negligible prior to ignition. If the onset time for the endothermic reaction is the shorter, then it can produce an asymptotic state in which a wide region of constant temperature (given approximately by the solution to $A e^{-E^{\prime} / \theta}=$ $E^{\prime}\left(\theta^{2}\right)$ and of ever increasing width exists at the surface of the solid, and this state will persist until reactant depletion diminishes the effect of the endothermic reaction. In this case, if the rate of the exothermic reaction is high enough to cause it to enter a transition stage before the endothermic reactant is appreciably depleted, then ignition will occur in a mechanism whereby the exothermic reaction undergoes a process akin to a classical homogeneous thermal explosion, at the asymptotic temperature of the endothermic reaction. Various other limits can be discussed; in all cases thermal runaway occurs first in a region that extends to the surface of the solid.

The finding, in Section 3, that during the transition stage a reactive-diffusive zone develops near the surface of the solid and a transient-diffusive zone in the interior, is remarkable because of the similarity to the behavior of other premixed reacting systems for large activation energies. For example, in steady propagation of premixed laminar flames, reactive-diffusive and convective-diffusive zones occur, in lowest order of a high activation-energy expansion. The convective-diffusive zone of the steady-state problem is entirely analogous to the transient-diffusive zone of the transient problem. The present result suggests that this kind of splitting may be quite universal in premixed combustion.

It is noteworthy that for this ignition problem, 
the asymptotic analysis yields accurate results over the entire range of parameters of physical interest. This suggests that asymptotic analysis may yield good results, without too great an expenditure of effort, for problems of greater complexity, which currently tax the calculational abilities of electronic computers when numerical methods are employed. For example, asymptotic methods might well produce analytical results for the complete ignition history, including transition to a steadily propagating deflagration, if reactant depletion and surface regression are included in the ignition analysis.

The work of the first author was partially supported by the Air Force Office of Scientific Research, through the European Office of the Office of Aerospace Research, United States Air Force, under Contract No. F61052-69-C-0036. The second author wishes to acknowledge fellowship support from the John Simon Guggenheim Memorial Foundation and additional support from the Air Force Office of Scientific Research, Office of Aerospace Research, United States Air Force under Project Themis Contract F44620-68-C-0010. We wish to thank Mr. V. Torroglosa for carrying out the computer calculation of $b_{0}$.

\section{APPENDIX A}

The purpose of this Appendix is to improve the rigor of the analysis, and also to investigate the effect of reactant consumption. We begin with the equations describing the temperature and concentration fields, as given by Bradley, viz.,

and

$$
\theta_{\tau}=\theta_{\xi \xi}+A(1-\varepsilon)^{a} \exp \left(-E^{\prime} / \theta\right)
$$

$$
\varepsilon_{\tau}=(A / B)(1-\varepsilon)^{a} \exp \left(-E^{\prime} / \theta\right),
$$

with the boundary conditions

$$
\theta_{\xi}(0, \tau)=-1, \quad \theta(\infty, \tau)=0
$$

and initial conditions

$$
\theta(\xi, 0)=1, \quad \varepsilon(\xi, 0)=0 .
$$

We seek the asymptotic solution of this system for large values of $E^{\prime}$, with the reaction order $a$, the nondimensional heat of reaction $B$ and the nondimensional inert temperature at ignition $\theta_{c}$ of order unity. We allow the solution to define the value of $A$ which produces thermal runaway at a given time $\tau_{c}$, assumed to be of order unity. For brevity of notation, we use as our small parameter

$$
\delta \equiv \theta_{c} / \sqrt{E^{\prime}}
$$

Working with the function $\Phi$, defined in equation (13), in place of $\theta$, we need to consider only the transition stage. With the inner and outer spatial variables $x$ and $\eta$, defined in equations (18) and (23), we anticipate the expansions

$$
\Phi=\delta^{2} \psi_{0}(x, \sigma)+\delta^{3} \psi_{1}(x, \sigma)+\delta^{4} \psi_{2}(x, \sigma)+\cdots
$$

and

$\Phi=\delta^{2} \Phi_{0}(\eta, \sigma)+\delta^{3} \Phi_{1}(\eta, \sigma)+\delta^{4} \Phi_{2}(\eta, \sigma)+\cdots$

for the inner, diffusive-reactive, and outer, transient-diffusive, layers. Here $\sigma$ is defined by the combination of equations (19) and (22), viz.,

$$
\sigma=\left(E^{\prime} / \theta_{c}^{2}\right)\left(\tau-\tau_{c}\right) / \sqrt{\pi \tau_{c}}+b_{0}
$$

where $b_{0}$, which we assume to be of order unity, is the first term of an expansion

of

$$
b=b_{0}+\delta b_{1}+\cdots,
$$

$$
b \equiv \ln \left[\left\{A \exp \left(-E^{\prime} / \theta_{c}\right)\right\}\left(\theta_{c}^{2} / E^{\prime}\right)^{1 / 2}\left(\pi \tau_{c}\right)^{1 / 4}\right] .
$$

We shall determine $b_{0}$ from the condition that in the first approximation for $\Phi$, thermal runaway occurs at $\sigma=b_{0}$; the following terms, $b_{1}$ etc., will be determined so as to minimize the singularities appearing at $\sigma=b_{0}$ in the higher-order approximations of $\Phi$. In the inner zone, the expansion of $\varepsilon$ is taken as

$$
\varepsilon=\delta \varepsilon_{1}(x, \sigma)+\delta^{2} \varepsilon_{2}(x, \sigma)+\cdots
$$

while in the outer zone $\varepsilon$ is anticipated to be exponentially small. For future use, we take note of the expansions

and

$$
\theta_{I}=\theta_{c}+\delta^{2}\left(\sigma-x-b_{0}\right)+0\left\{\delta^{4}\right\}
$$

$$
\theta_{I}=\theta_{c}-\delta\left(\pi \tau_{c}\right)^{1 / 4} \eta+0\left\{\delta^{2}\right\}
$$

which follow from equations (7), (18), (23) and (A8).

Use of equations (18), (A6) and (A8) through (A12) in equations (AI) and (A2) produces the inner equations

$$
\begin{aligned}
& \psi_{0 x x}+\delta \psi_{1 x x}+\delta^{2} \psi_{2 x x}-\delta^{2}\left(\pi \tau_{c}\right)^{-1 / 2} \psi_{0 \sigma}+0\left\{\delta^{3}\right\}=R \\
& \quad \equiv-\delta\left(\pi \tau_{c}\right)^{-1 / 4}\left[1-\delta \varepsilon_{1}+0\left\{\delta^{2}\right\}\right]^{a} \\
& \quad \times \exp \left[\sigma-x+\psi_{0}+\delta \psi_{1}+\delta b_{1}+0\left\{\delta^{2}\right\}\right] \quad \text { (A14) }
\end{aligned}
$$

and

$$
\varepsilon_{1 \sigma}+\delta \varepsilon_{2 \sigma}+0\left\{\delta^{2}\right\}=-B^{-1}\left(\pi \tau_{c}\right)^{1 / 2} R / \delta
$$


The initial conditions for these equations are found from equation (A4) to be

$$
\begin{array}{ll}
\psi_{j}(x,-\infty)=0, & j=0,1,2, \ldots \\
\varepsilon_{j}(x,-\infty)=0, & j=1,2,3, \ldots
\end{array}
$$

Equation (A3) produces the boundary condition

$$
0=\psi_{j x}(0, \sigma), \quad j=0,1,2 \cdots
$$

When equations (23), (A7), (A8), (A9), (A10) and (A13) are used in equations (A1) and (A2), one obtains as the outer equation

$$
\begin{gathered}
\Phi_{0 \sigma}-\Phi_{0 \eta \eta}+\delta\left(\Phi_{1 \sigma}-\Phi_{1 \eta \eta}\right)+0\left\{\delta^{2}\right\}= \\
\left(\pi \tau_{e}\right)^{1 / 4} \delta^{-1} \exp \left\{-\left(\pi \tau_{c}\right)^{1 / 4} \delta^{-1} \eta+0(1)\right\}
\end{gathered}
$$

with boundary and initial conditions

$$
\Phi_{j}(\infty, \sigma)=\Phi_{j}(\eta,-\infty)=0, j=0,1,2, \ldots
$$

as implied by equations (A3) and (A4). The equation and initial condition obtained for $\varepsilon$ in the outer zone verify that $\varepsilon$ is exponentially small for any value of $\eta$ of order unity or greater.

Expressed in the outer variable, the matching conditions, obtained from equations (18), (23), (A6) and (A7), are

$$
\begin{aligned}
\lim _{\eta \rightarrow 0}\left\{\Phi_{0}(\eta, \sigma)\right. & \left.+\delta \Phi_{1}(\eta, \sigma)+\cdots\right\} \\
\sim & \lim _{\eta \rightarrow \infty}\left[\psi_{0}\left\{\left(\pi \tau_{c}\right)^{1 / 4} \delta^{-1} \eta, \sigma\right\}+\delta \psi_{1}\left\{\left(\pi \tau_{c}\right)^{1 / 4} \delta^{-1} \eta, \sigma\right\}\right. \\
& \left.\quad+\delta^{2} \psi_{2}\left\{\left(\pi \tau_{c}\right)^{1 / 4} \delta^{-1} \eta, \sigma\right\}+\cdots\right] \quad(\mathrm{A} 20)
\end{aligned}
$$

which should, in fact, be rewritten in terms of a parametric limit process performed in an intermediate variable to see most clearly the proper ordering.

In the inner zone, to lowest order, equation (A14) implies $\psi_{0 x x}=0$, whose solution consistent with equation (A17) is

$$
\psi_{0}=\psi_{0}(\sigma)
$$

where $\psi_{0}(\sigma)$ is a function of $\sigma$ to be determined from the matching conditions. Equation (A15) then yields

$$
\varepsilon_{1}=B^{-1}\left(\pi \tau_{c}\right)^{1 / 4} e^{-x} \int_{-\infty}^{\sigma} \exp \left[\sigma+\psi_{0}(\sigma)\right] d \sigma
$$

in view of equation (A16). To the next order, equation (A 14) reduces to

$$
\psi_{1 x x}=-\left(\pi \tau_{c}\right)^{-1 / 4} \exp \left[\sigma-x+\psi_{0}(\sigma)\right]
$$

whose solution satisfying equation (A17) is

$$
\psi_{1}=-\left(\pi \tau_{c}\right)^{-1 / 4}\left[x+e^{-x}+f_{1}(\sigma)\right] \exp \left[\sigma+\psi_{0}(\sigma)\right]
$$

where $f_{1}(\sigma)$ is a function of $\sigma$ to be determined from the matching conditions. Higher-order inner equations are seen to be qualitatively similar to equations (A22), (A23) and (A24), although somewhat more complicated algebraically.

Matching requirements between the two-term inner expansion and the one-term outer expansion, derived from equation (A20), give

and

$$
\Phi_{0}(0, \sigma)=\psi_{0}(\sigma)
$$

$$
\Phi_{0 \eta}(0, \sigma)=-\exp \left\{\sigma+\psi_{0}(\sigma)\right\}
$$

where use has been made of equations (A21) and (A24). As often occurs in matching procedures, a number of higher-order matching requirements are satisfied automatically.

When $\psi_{0}(\sigma)$ is eliminated from equation (A26) by use of equation (A25), one obtains from the term of lowest order in equation (A18), supplemented by equation (A19) for $j=0$, precisely the same problem for $\Phi_{0}(\eta, \sigma)$ that was defined in equation (28). This justifies, to lowest order, the heuristic approach adopted in the main text. It also reveals that to this order reactant depletion does not affect the ignition time. The time history of the fraction of the reaction completed at the surface, normalized through multiplication by the large parameter $\delta^{-1} B\left(\pi \tau_{c}\right)^{-1 / 4}$, as predicted by equation (A22), is shown in Figure 1, from which it can be seen that $\varepsilon_{1}$ approaches a finite limit at thermal runaway, a result which can also be deduced analytically from an asymptotic analysis for large $\Phi_{0}(0, \sigma)$, by using equation (B4). The extent of reactant consumption at the moment of ignition, calculated from the limit of $\varepsilon_{1}$, for the range of parameters considered by Bradley (1970), agrees within $\pm 40 \%$ with Bradley's equation (9), which he states correlates his numerical results for reactant depletion within $10 \%$; our result differs somewhat functionally from that of Bradley's correlation formula, the ratio of our depletion to his being $2.6 \theta_{c} \tau_{e}^{-1 / 4} / \sqrt{E^{\prime}}$. As might be inferred from the $\varepsilon$ curve in the insert in Fig. 1, the extent of reactant consumption at ignition is much more strongly dependent on the choice of ignition criterion than is the ignition time. Equations (A14) and (A22) show that our reactant depletion will influence 
$\psi_{2}$, through a term proportional to $a B^{-1}$; it follows that low reaction order and high heat of reaction both tend to minimize the effects of reactant depletion.

\section{APPENDIX B}

To integrate equation (28) numerically, we first convert the problem to an integral equation in one variable. By transform methods or Green's functions, it can be shown from equation (28) that $\psi(0, \sigma)$ obeys the integral equation

$$
\begin{array}{r}
\psi(0, \sigma)=(2 / \sqrt{\pi}) \int_{-\infty}^{\sigma} \sqrt{\sigma-\tau}\left\{1+\psi_{\tau}(0, \tau)\right\} \\
\times \exp \{\psi(0, \tau)+\tau\} d \tau
\end{array}
$$

In equation (B1) the integral from $-\infty$ to a large negative value $\sigma_{0}$ can be evaluated analytically, and equation (B1) then becomes

$$
\begin{aligned}
\psi(0, \sigma)= & e^{\sigma}\left\{(2 / \sqrt{\pi}) \sqrt{\sigma-\sigma_{0}} \exp \left\{-\left(\sigma-\sigma_{0}\right)^{2}\right\}\right. \\
& \left.+\operatorname{erfc}\left(\sqrt{\sigma-\sigma_{0}}\right)\right\} \\
& +(2 / \sqrt{\pi}) \int_{\sigma_{0}}^{\sigma} \sqrt{\sigma-\tau} \exp \{\psi(0, \tau)+\tau\} \\
& \times\{d \tau+d \psi(0, \tau)\}
\end{aligned}
$$

A suitable finite-difference version of equation
(B2) is

$$
\begin{aligned}
\psi_{j}= & \exp \left(\sigma_{j}\right)\left\{(2 / \sqrt{\pi}) \sqrt{\sigma_{j}-\sigma_{0}} \exp \left\{-\left(\sigma_{j}-\sigma_{0}\right)^{2}\right\}\right. \\
& \left.+\operatorname{erfc}\left(\sqrt{\sigma_{j}-\sigma_{0}}\right)\right\} \\
& +\frac{2}{\sqrt{\pi}} \sum_{i=1}^{j-1}\left(\sigma_{j}-\frac{\sigma_{i}+\sigma_{i+1}}{2}\right)^{1 / 2} \\
& \times \exp \left\{\frac{1}{2}\left(\psi_{i}+\psi_{i+1}+\sigma_{i}+\sigma_{i+1}\right)\right\} \\
& \times\left(\psi_{i+1}-\psi_{i}+\sigma_{i+1}-\sigma_{i}\right)
\end{aligned}
$$

In equation (B3) a series of values for $\psi_{j}$ was selected, and the resulting algebraic equations for $\sigma_{j}$ were solved sequentially by a NewtonRaphson iterative procedure, thus generating the function $\psi(0, \sigma)$. This function was used for performing the integration in equation (A22) numerically to obtain $\varepsilon_{1}(0, \sigma)$.

The numerical integration yielded $b_{0}=-0.431$. For large values of $\psi(0, \sigma)$, the numerical results agree well with an asymptotic expression that can be derived analytically from equation (28) by treating $\exp \{-\psi(0, \sigma)\}$ as the dependent variable. The asymptotic expression is

$$
\begin{gathered}
\psi(0, \sigma) \simeq-\frac{1}{2} \ln \left(b_{0}-\sigma\right)-b_{0}-\frac{1}{2} \ln 2 \\
\text { REFERENCE }
\end{gathered}
$$

Bradley, H. H., Jr. 1970. Theory of Ignition of a Reactive Solid by Constant Energy Flux, Comb. Sci. and Tech. 2, 11.

Received February 16, 1971. 